\title{
Blocking aggrecanase cleavage in the aggrecan interglobular domain abrogates cartilage erosion and promotes cartilage repair
}

\author{
Christopher B. Little,,1,2 Clare T. Meeker, ${ }^{1}$ Suzanne B. Golub, ${ }^{1}$ Kate E. Lawlor, ${ }^{1}$ Pamela J. Farmer,1,3 \\ Susan M. Smith, ${ }^{2}$ and Amanda J. Fosang ${ }^{1}$ \\ ${ }^{1}$ Arthritis Research Group, University of Melbourne Department of Paediatrics and Murdoch Childrens Research Institute, Royal Children's Hospital, Parkville, \\ Victoria, Australia. ${ }^{2}$ Raymond Purves Bone and Joint Research Laboratories, University of Sydney at the Royal North Shore Hospital, St. Leonards, \\ New South Wales, Australia. ${ }^{3}$ Surgical Research, Murdoch Childrens Research Institute, Royal Children's Hospital, Parkville, Victoria, Australia.
}

\begin{abstract}
Aggrecan loss from cartilage in arthritis is mediated by aggrecanases. Aggrecanases cleave aggrecan preferentially in the chondroitin sulfate-2 (CS-2) domain and secondarily at the $\mathrm{E}^{373} \downarrow^{374} \mathrm{~A}$ bond in the interglobular domain (IGD). However, IGD cleavage may be more deleterious for cartilage biomechanics because it releases the entire CS-containing portion of aggrecan. Recent studies identifying aggrecanase-2 (ADAMTS-5) as the predominant aggrecanase in mouse cartilage have not distinguished aggrecanolysis in the IGD from aggrecanolysis in the CS-2 domain. We generated aggrecan knockin mice with a mutation that rendered only the IGD resistant to aggrecanases in order to assess the contribution of this specific cleavage to cartilage pathology. The knockin mice were viable and fertile. Aggrecanase cleavage in the aggrecan IGD was not detected in knockin mouse cartilage in situ nor following digestion with ADAMTS-5 or treatment of cartilage explant cultures with IL-1 $\alpha$. Blocking cleavage in the IGD not only diminished aggrecan loss and cartilage erosion in surgically induced osteoarthritis and a model of inflammatory arthritis, but appeared to stimulate cartilage repair following acute inflammation. We conclude that blocking aggrecanolysis in the aggrecan IGD alone protects against cartilage erosion and may potentiate cartilage repair.
\end{abstract}

\section{Introduction}

In joint pathology, the loss of aggrecan from articular cartilage is a proteolytic process driven predominantly by aggrecanases. One aggrecanase cleavage site is located in the interglobular domain (IGD) near the $\mathrm{N}$ terminus, and 4 other sites are located in the chondroitin sulfate-2 (CS-2) domain at the $\mathrm{C}$ terminus. Aggrecanase was first identified as a novel activity that cleaves the aggrecan core protein at the $E^{373} \downarrow^{374} \mathrm{~A}$ bond in the IGD (1-3), and its activity was simultaneously identified in synovial fluids from osteoarthritic joint injury and inflammatory joint disease patients $(4,5)$. The aggrecanases are members of the ADAMTS (a disintegrin and metalloproteinase with thrombospondin motifs) family and are designated aggrecanase-1 (ADAMTS-4; ref. 6) and aggrecanase-2 (ADAMTS-5; ref. 7). ADAMTS enzymes contain a zinc-dependent catalytic domain consensus sequence, HExxHxxGxxH, where $\mathrm{H}$ (histidine) represents strictly conserved zinc ligands. There is a conserved methionine residue located approximately 20 amino acids C-terminal to the third histidine, which forms a met-turn. Following the catalytic domain, ADAMTS enzymes have a disintegrin domain, 1 or more thrombospondin motifs, a cysteine-rich domain, and a spacer domain of variable

Nonstandard abbreviations used: ADAMTS, a disintegrin and metalloproteinase with thrombospondin motifs; AIA, antigen-induced arthritis; CS, chondroitin sulfate; $\Delta$ di-4S, $\Delta$-disaccharide of CS-4; DMM, destabilization of the medial meniscus; IGD, interglobular domain.

Conflict of interest: A.J. Fosang received financial support from Pfizer Inc. for this study.

Citation for this article: J. Clin. Invest. 117:1627-1636 (2007). doi:10.1172/JCI30765 length (8-10). ADAMTS-5 has recently been identified as the major aggrecanase in mouse cartilage $(11,12)$.

Cleavage at the $\mathrm{E}^{373} \downarrow^{374} \mathrm{~A}$ bond is considered the signature activity of the aggrecanases, not only because it was the site first characterized, but also because the ITEGE ${ }^{373}$ neoepitope has been widely reported in humans and animals both in vitro and in vivo $(8,10$, 13 , 14). However, cleavage at $\mathrm{E}^{373} \downarrow^{374} \mathrm{~A}$ is not the preferred action of these enzymes. Results of in vitro studies with human recombinant ADAMTS-4 (15) and ADAMTS-5 (16) have shown that they preferentially cleave aggrecan in the CS-2 domain. The 2 most preferred cleavage sites in bovine aggrecan are at KEEE ${ }^{1666} \downarrow{ }^{1667}$ GLGS and GELE ${ }^{1480} \downarrow{ }^{1481}$ GRGT. Subsequently, further cleavages can occur at TAQE ${ }^{1771} \downarrow^{1772} \mathrm{AGEG}$ and VSQE ${ }^{1871} \downarrow^{1872} \mathrm{LGQR}$ in the CS-2 region and at ITEGE ${ }^{373} \downarrow^{374} \mathrm{ARGSV}$ in the IGD. A similar pattern of cleavage site preferences is seen in the rat chondrosarcoma (17).

Although cleavage occurs preferentially in the CS- 2 domain, it only releases a proportion of the glycosaminoglycan chains essential for weight bearing and may therefore be less critical for the functional and biomechanical properties of cartilage. Conversely, cleavage in the IGD releases the entire glycosaminoglycan-containing (CS and keratan sulfate) portion of aggrecan and may have the most deleterious effects. Because few experiments to test these tenets have been done, the significance of regional aggrecanolysis in the core protein remains unclear. In vitro testing of cartilage explants in confined compression experiments has shown that cleavage in the IGD correlates with a loss of mechanical properties, whereas cleavage at sites in the CS-2 domain do not appear to affect biomechanics (18). There have been no in vivo studies to date. 
A

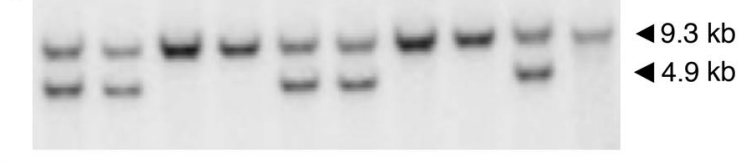

B

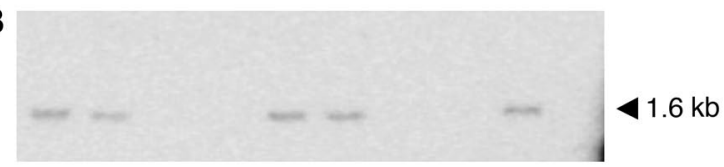

C

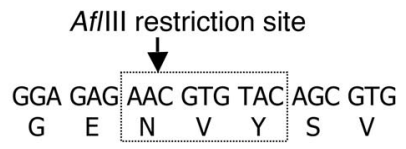

D

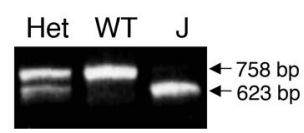

We and others recently examined mice deficient in ADAMTS-5 activity and showed that ablation of ADAMTS- 5 activity protects against aggrecan loss and cartilage erosion in inflammatory (12) and noninflammatory (11) models of arthritis. Thus ADAMTS-5 appears to be the major aggrecanase in the mouse, and specific inhibitors of ADAMTS- 5 activity are in development for cartilagepreserving therapeutics $(19,20)$. However, the in vivo studies with ADAMTS-5 deficient mice $(11,12)$ did not distinguish between aggrecanolysis in the IGD, and aggrecanolysis at the preferred sites in the CS-2 domain.

In the present study, we generated genetically modified mice with a mutation in the aggrecan core protein that rendered the IGD resistant to aggrecanases. These mice, which resist aggrecanase cleavage at the $\mathrm{E}^{373} \downarrow^{374} \mathrm{~A}$ site in the IGD but remain susceptible to cleavage at sites in the CS- 2 domain, provided an opportunity to analyze the contribution of IGD cleavage to cartilage pathology in vivo. We report here that resistance to aggrecanase cleavage in the IGD alone conferred significant protection against the development of cartilage lesions in both inflammatory and noninflammatory models of murine arthritis. Furthermore, mice resistant to aggrecanolysis in the IGD reestablish toluidine blue staining in aggrecan-depleted cartilage, which suggested that blocking IGD cleavage may accelerate cartilage repair. Overall, the data suggest that aggrecanase inhibitors targeting the aggrecan IGD may protect against cartilage erosion and potentiate cartilage repair.

\section{Results}

We created C57BL/6 aggrecan knockin mice with a targeted mutation in exon 7 . The mutation changed amino acids ${ }^{374} \mathrm{ALGS}$ to ${ }^{374} \mathrm{NVYS}$ and was designed to eliminate aggrecanase cleavage at the $E^{373} \downarrow^{374} \mathrm{~A}$ bond

\section{Figure 2}

\section{Figure 1}

Mutation detection by Southern blotting and PCR. (A) Southern blotting of genomic DNA detected 9.3- and 4.9-kb BamHI fragments in wild-type and Jaffa alleles, respectively. (B) After stripping, a 1.6-kb fragment was detected in Jaffa DNA on the same filter probed with a neo cDNA probe. (C) The mutation that changed amino acids ${ }^{374} \mathrm{ALG}$ to ${ }^{374} \mathrm{NVY}$ introduced a unique AfIIII restriction site in exon 7. (D) A 758bp PCR fragment amplified from wild-type DNA was resistant to AfIIII digestion. AfIIII digestion of the PCR fragment amplified from Jaffa (J) and heterozygote (Het) DNA yielded 623-bp and 135-bp fragments, although the 135-bp fragment was rarely visible on the gels.

in vitro (21) and in vivo. Mice homozygous for the mutation were designated Jaffa mice, and their genotypes were confirmed by Southern blotting of genomic DNA. An aggrecan cDNA probe detected BamHI fragments corresponding to the wild-type $(9.3 \mathrm{~kb})$ and Jaffa $(4.9 \mathrm{~kb})$ alleles (Figure 1A). After stripping the filters, a neo cDNA probe detected a $1.6-\mathrm{kb}$ fragment in mice bearing the mutation (Figure 1B). The mutation changing amino acids ${ }^{374} \mathrm{ALG}$ to ${ }^{374} \mathrm{NVY}$ introduced a unique AflIII restriction site in exon 7 (Figure 1C). For routine genotyping, genomic DNA was extracted from tail biopsies and analyzed by PCR amplification of a 758-bp cDNA fragment. AflIII digestion yielded 623- and 135-bp fragments from Jaffa DNA and 758-, 623-, and 135-bp fragments from heterozygous DNA, although the 135-bp fragment was not routinely detected. Only the undigested, 758-bp fragment was detected in wild-type DNA lacking the AflIII restriction site (Figure 1D). Heterozygous breeding pairs produced normal-sized litters with the expected ratio of homozygous wild-type and Jaffa pups (28\% and $24 \%$, respectively, from 202 pups in 35 litters), with no significant difference in growth

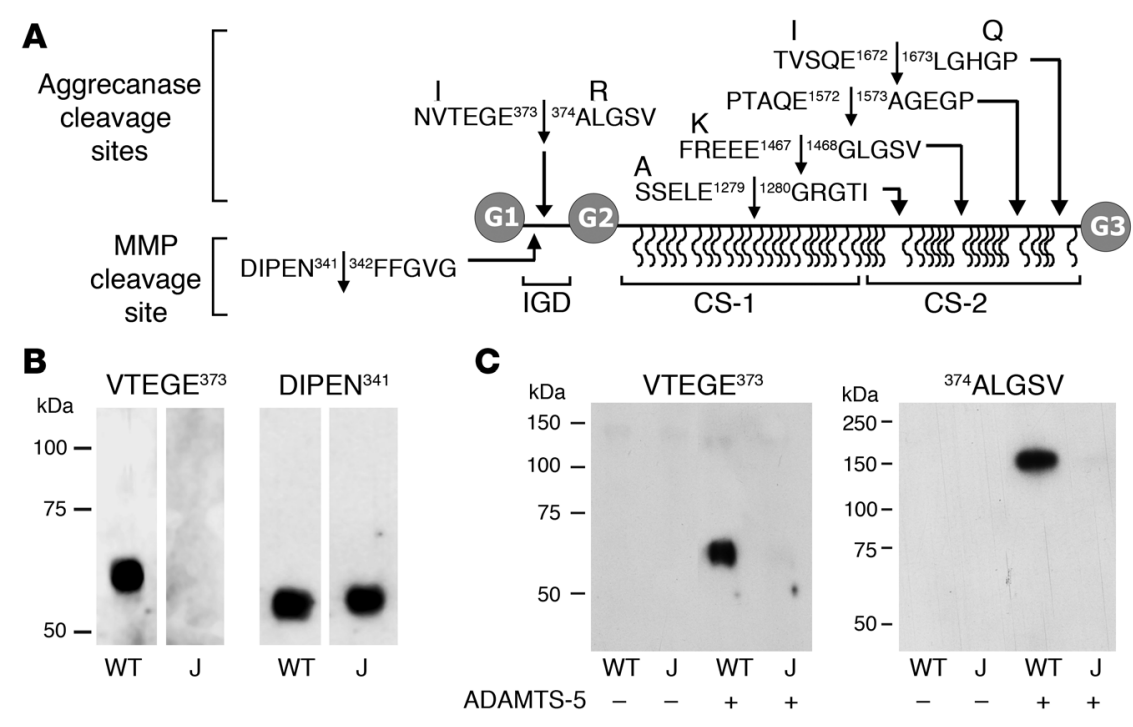

G1 fragments in Jaffa and wild-type cartilage extracts. (A) The domain structure of the aggrecan core protein, with $2 \mathrm{~N}$-terminal globular domains, G1 and G2, and a C-terminal G3 domain. The extended region between G2 and G3 is heavily substituted with CS chains (wavy lines). Aggrecanase and MMP cleavage site sequences in the mouse IGD and CS-2 domains are shown, and amino acids that are different in human aggrecan are shown above. Numbering corresponds with the mouse sequence. (B) Cartilage extracts from wild-type and Jaffa mice were analyzed for VTEGE 373 or DIPEN ${ }^{341}$ neoepitopes. (C) Dialyzed extracts of equal cartilage wet weight from wild-type and Jaffa mice were digested with or without recombinant human ADAMTS-5 and analyzed for the VTEGE ${ }^{373}$ and ${ }^{374}$ ALGSV neoepitopes. Stripping and reprobing the membranes with monoclonal antibody 2B6 confirmed sample loading in each lane (data not shown). 
A Newborn $12 \mathrm{~d}$

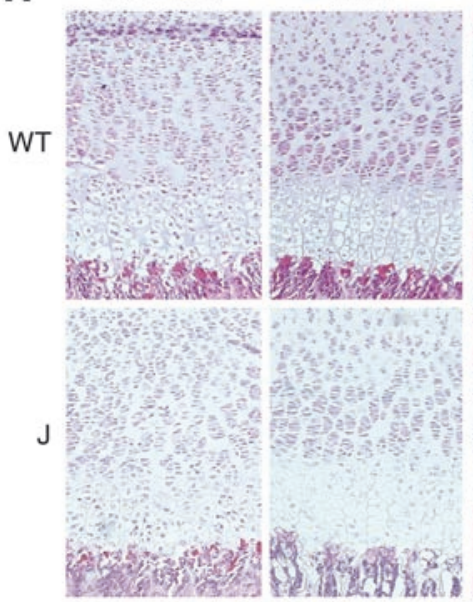

B

WT
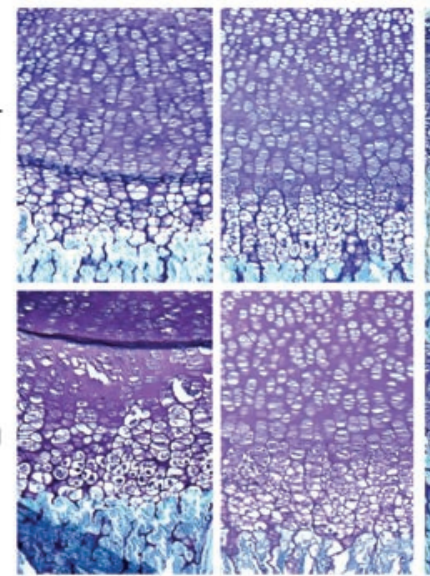

3 wk
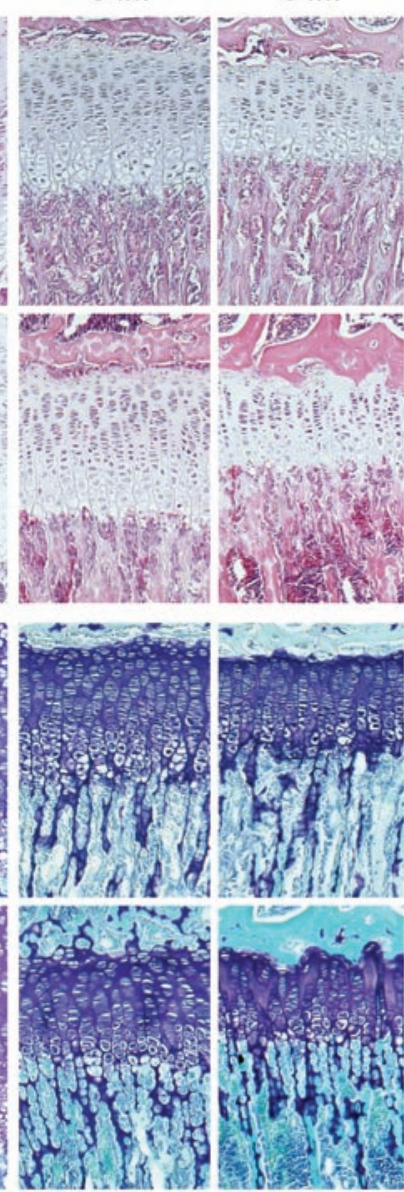

6 wk
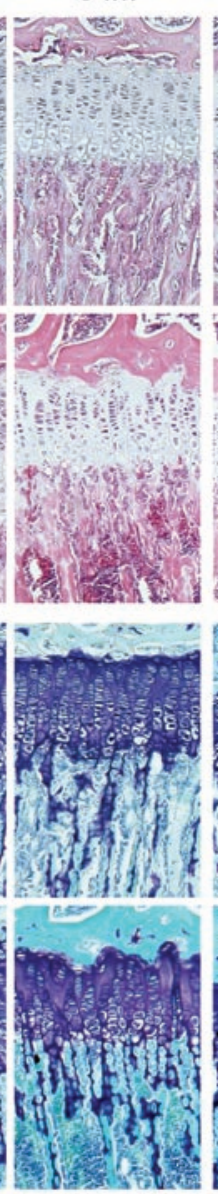

$8 w k$
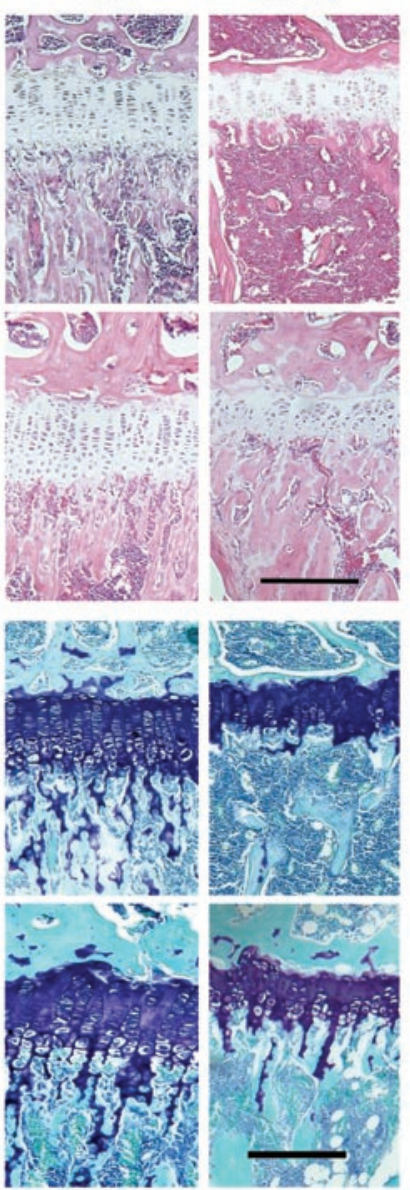

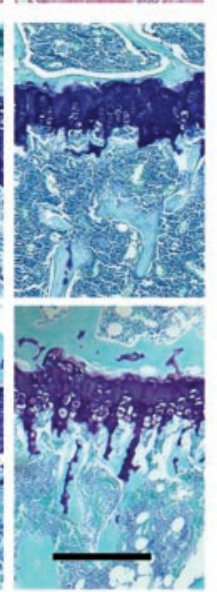

\section{Figure 3}

Jaffa has normal growth plate morphology and aggrecan content. Paraffin sections of wild-type and Jaffa proximal tibial growth plates from birth to 13 weeks of age were stained with H\&E (A) or toluidine blue (B). There was no difference in growth plate architecture between genotypes at any age. Scale bars: $200 \mu \mathrm{m}$.

Skeletal growth and cartilage architecture are normal in Jaffa mice. Homozygous Jaffa mice were phenotypically normal. Standard histological analysis of major nonskeletal tissues (heart, brain, kidney, and lung) with $\mathrm{H} \& \mathrm{E}$ staining failed to reveal any morphological differences between Jaffa mice and wild-type controls (data not shown). Furthermore, there was no difference in the skeletal morphology of Jaffa mice compared with wild-type mice at any age between E16.5 and 12 months. Representative proximal tibial growth plates stained with $\mathrm{H} \& \mathrm{E}$ or toluidine blue from Jaffa and wild-type mice at birth, 12 days, and 3, 6, 8, and 13 weeks are shown in Figure 3, A and B. There were no detectable differ-

rates (data not shown). Homozygous Jaffa breeders were fertile and had litters of normal size and gender distribution.

Jaffa aggrecan is resistant to ADAMTS cleavage in the IGD. Neoepitope antibodies recognize newly created $\mathrm{N}$ - or $\mathrm{C}$-terminal sequences on polypeptide fragments generated by proteolysis, but fail to recognize the same sequence of amino acids in the intact core protein $(13,22)$. Neoepitope antibodies can therefore be used to identify products of specific cleavage events, and antibodies against the major aggrecan neoepitopes generated by aggrecanases and MMPs (Figure 2A) have been well characterized (12, 23, 24). We used neoepitope Western blotting to confirm that aggrecan from Jaffa cartilage was resistant to aggrecanase cleavage in the IGD. The VTEGE ${ }^{373}$ neoepitope was readily detected in extracts of wildtype cartilage but absent from Jaffa cartilage (Figure 2B), indicating that no in vivo cleavage occurred at the aggrecanase site in Jaffa mice. The amount of DIPEN ${ }^{341}$ neoepitope generated by MMP cleavage in the IGD was the same in wild-type and Jaffa mice (Figure $2 \mathrm{~B}$ ), indicating that in vivo, MMPs do not compensate for the lack of aggrecanase cleavage in Jaffa.

To further confirm that Jaffa aggrecan was resistant to aggrecanase cleavage in the IGD, dialyzed cartilage extracts were incubated with recombinant ADAMTS-5 and analyzed for aggrecanase neoepitopes. The VTEGE ${ }^{373}$ and ${ }^{374}$ ALGSV neoepitopes were readily detected in wild-type aggrecan but were not generated by ADAMTS-5 digestion of Jaffa aggrecan (Figure 2C). This result confirms that the IGD mutation confers resistance to aggrecanases. ences between the genotypes at any age. The width of tibial growth plates, chondrocyte distribution, and chondrocyte morphology did not vary with genotype, and there was no apparent accumulation of aggrecan, as assessed by toluidine blue staining. The normal architecture of the Jaffa growth plate strongly suggests that aggrecanase cleavage in the IGD is not required for aggrecan resorption during endochondral ossification or skeletal growth.

We postulated that aggrecan turnover might have been reduced in Jaffa mice, leading to aggrecan accumulation in Jaffa cartilage. However, the aggrecan content in femoral heads from 3- to 9-weekold wild type and Jaffa mice was $24.7 \pm 1.1$ and $24.6 \pm 1.7 \mu$ g sulfated glycosaminoglycan/mg tissue wet wt (mean $\pm \mathrm{SD}$ ), respectively. This result shows that blocking aggrecanase cleavage in the IGD did not lead to aggrecan accumulation in developing or mature cartilage and was consistent with the uniformity of the toluidine blue staining in Figure 3B.

To eliminate the possibility that an accumulation of aggrecan in Jaffa mice might not be detected in the 1,9 dimethylmethylene blue assay if Jaffa aggrecan was substantially undersulfated, we analyzed the $\Delta$-disaccharides of CS by fluorophore-assisted carbohydrate electrophoresis. We found that in the cartilage of 10-dayold mice, the $\Delta$-disaccharide of CS- 4 ( $\Delta$ di- $4 \mathrm{~S})$ was the predominant species, and whereas $\Delta$ di-0S was present in low amounts, $\Delta$ di-6S and $\Delta$ di-2S were undetectable (data not shown). The content of $\Delta$ di-4S and $\Delta$ di-0S in Jaffa and wild-type cartilage was the same, indicating that there was no difference in the type or extent of sul- 

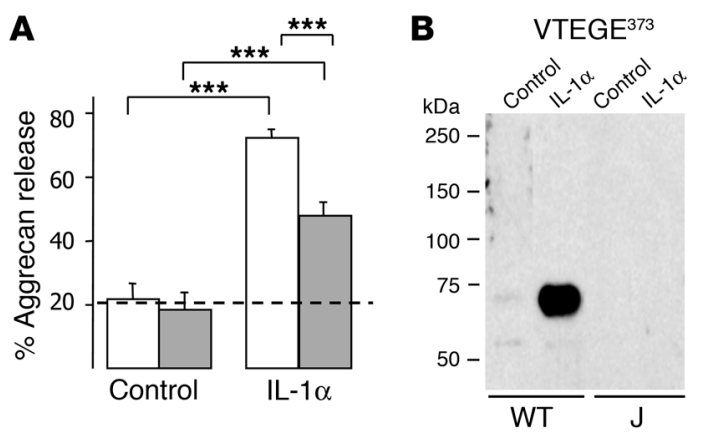

Figure 4

Aggrecan degradation in vitro. (A) Aggrecan release from wild-type (white bars) and Jaffa (gray bars) cartilage cultured with or without $10 \mathrm{ng} / \mathrm{ml} \mathrm{IL-1 \alpha}$ ( $n=8-10$ per treatment). Results are mean \pm SEM. The dotted line shows the level of baseline release. ${ }^{* * *} P<0.001$. (B) Cartilage extracts from wild-type and Jaffa mice with or without IL-1 $\alpha$ treatment were analyzed by Western blot. Membranes were immunolocalized for VTEGE ${ }^{373}$ neoepitope.

fation on CS chains in Jaffa compared with wild-type mice and further demonstrating that there was no accumulation of aggrecan in Jaffa. Identical results were found in the Chloe mouse, in which the IGD sequence DIPEN ${ }^{341} \downarrow^{342}$ FFGVG was mutated to DIPEN $^{341 / 342}$ GTRVG, rendering Chloe aggrecan resistant to IGD cleavage by MMPs (25).

Aggrecan degradation in vitro. Our primary interest in Jaffa is the extent to which aggrecanolysis in this mouse can be curtailed by selectively blocking cleavage at the IGD site only both in vitro and in vivo. In vitro experiments were done with femoral head cartilage explants with and without treatment with IL-1 $\alpha$ to drive aggrecanolysis (Figure 4). The amount of aggrecan released from unstimulated cultures at baseline was the same in wild-type and Jaffa cartilage $(21.8 \% \pm 5 \%$ and $18.5 \% \pm 5 \%$, respectively; Figure $4 \mathrm{~A})$. Whereas IL-1 $\alpha$ increased aggrecan loss from both wild-type and Jaffa cartilage, the amount of aggrecan released from Jaffa cartilage was significantly less than that from wild-type cartilage $(P<0.001$ for all comparisons; Figure 4A). The aggrecan loss relative to baseline was $41 \%$ less from Jaffa than from wild-type mice, consistent with the fact that glycosaminoglycan-containing fragments continue to be released from Jaffa cartilage by aggrecanase cleavage in the CS-2 domain even though cleavage in the IGD is blocked. The aggrecan loss induced by IL-1 $\alpha$ treatment was mediated by aggrecanases and generated VTEGE ${ }^{373}$ neoepitope in wildtype-cartilage, whereas no VTEGE ${ }^{373}$ fragments were created in Jaffa cartilage (Figure 4B). There was no compensatory increase in the DIPEN ${ }^{341}$ neoepitope in Jaffa cartilage (data not shown).

These in vitro results confirmed that the aggrecan knockin mutation blocked aggrecanolysis in the IGD, and Figure 4A shows that as predicted, the extent of aggrecan release from cartilage was partly, but not fully, diminished. Next, to assess the in vivo consequence of blocking aggrecanolysis in the IGD, we induced experimental arthritis in mice using inflammatory and noninflammatory models. For these experiments we included a strain of C57BL/6 mouse that is resistant to IGD cleavage at the MMP cleavage site for comparison. Like the Jaffa mouse, the Chloe mouse is phenotypically normal (25).

Osteoarthritis-like cartilage lesions are less severe in Jaffa mice. As previously reported, destabilization of the medial meniscus (DMM) in mice causes progressive osteoarthritis-like disease with focal aggrecan loss and structural damage to articular cartilage with little or no synovial inflammation (26). The model produces focal lesions, and analyses are done by serial sections across the entire width of the joint. Serial sections from any 1 joint showed variations in lesion severity, as demonstrated by the representative mild, moderate, and severe lesions from wild-type mice shown in Figure 5, A-C. Because the lesions were focal and variable, the results are presented as the sum of scores for all sections in order to account for both the severity and the spread of the lesion. We found that cartilage damage in the tibia was more severe and more consistent than it was in the femur; therefore, only changes in the tibia are reported here.

Four weeks after surgical destabilization, the extent of aggrecan loss from wild-type and Chloe cartilage was similar, but aggrecan loss from Jaffa cartilage was significantly less $(31 \%-45 \% ; P<0.05$ for all comparisons). By 8 weeks, aggrecan loss had progressed in all strains $(P<0.01$ for all comparisons) such that there was no difference between Jaffa and wild-type cartilage (data not shown). These results suggest that Jaffa is protected against aggrecan loss at early but not late time points in this model.

The most interesting results were the differences seen in structural damage to Jaffa, Chloe, and wild-type cartilage (Figure 5D). By 4 weeks, the structural damage to wild-type and Chloe cartilage was similar; however, the extent of structural damage to Jaffa cartilage was approximately $40 \%$ less, suggesting that the Jaffa mouse was protected from overt cartilage degeneration at this time point.

\section{Figure 5}

DMM model in wild-type and aggrecan knockin mice. (A-C) Representative toluidine blue/fast green-stained sagittal sections of medial femorotibial joints from wild-type mice demonstrating mild (A), moderate (B), and severe lesions (C). Scale bar: $200 \mu \mathrm{m}$. (D) Sum of scores for tibial cartilage structural damage (mean \pm SEM) in the medial tibial plateau at 4 or 8 weeks after surgery in wild-type $(n=12$ [4 wk], 11 [8 wk]; open bars), Chloe ( $n=13$ [4 wk], 11 [8 wk]; black bars), and Jaffa ( $n=11$ [4 wk], 8 [8 wk]; gray bars) joints after DMM induction. ${ }^{* \star} P<0.01 ;{ }^{* *} P<0.001$.
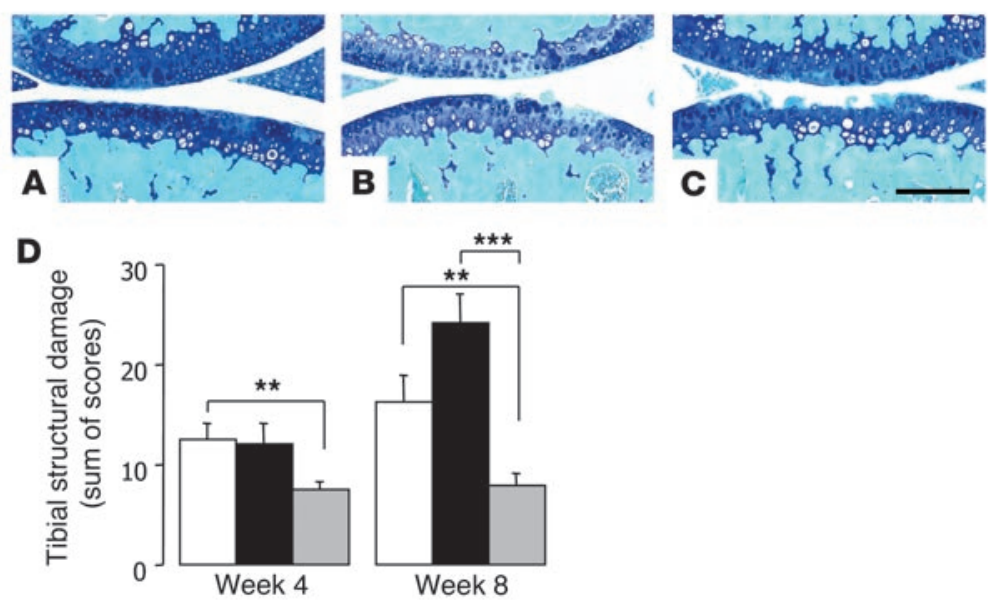
A

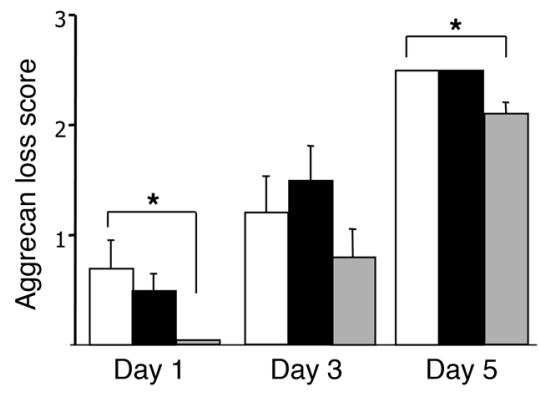

B
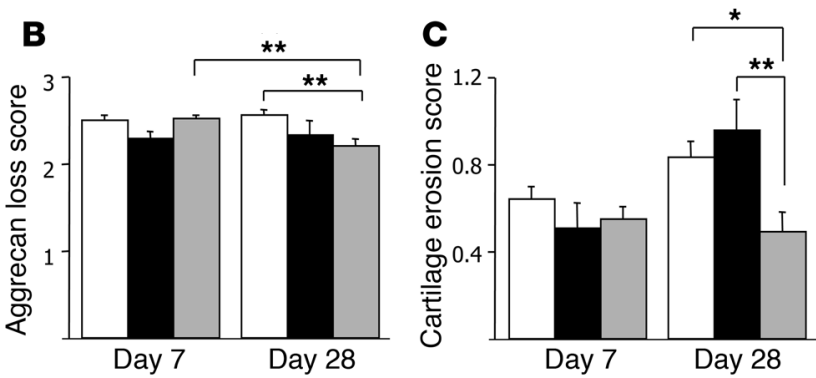

By 8 weeks, protection in Jaffa cartilage was more pronounced, with the extent of damage $52 \%$ and $67 \%$ less than in wild-type and Chloe cartilage, respectively ( $P<0.01$ for all comparisons). Overall, these results demonstrate that Jaffa is protected against the progressive osteoarthritis-like degeneration of articular cartilage following DMM surgery. There were no differences in the proportion of TUNEL-positive cells at 4 or 8 weeks after DMM.

Cartilage erosions in inflammatory arthritis are less severe in Jaffa mice. We next compared cartilage pathology using antigen-induced arthritis (AIA), a model of monoarticular inflammatory disease. Initially we monitored early, acute disease and found that synovitis and exudation into the joint cavity were similar in all genotypes on days 1,3 , and 5 (data not shown). This was not surprising, as the knockin mutation was not predicted to affect inflammation. Instead we predicted that aggrecan loss might have been reduced in Jaffa mice at early time points because ADAMTS-5-deficient mice were protected against aggrecan loss at day 3 in the AIA model (12). We found that aggrecan loss from wild-type, Chloe, and Jaffa cartilage increased progressively during the acute phase of the disease and that aggrecan loss from Jaffa cartilage was indeed less than that from wild-type and Chloe cartilage on days 1, 3, and 5; the difference between Jaffa and wild-type cartilage was significant on days 1 and 5 ( $P<0.05$; Figure $6 \mathrm{~A})$.

After the first-week inflammatory flare in the AIA model, inflammation gradually subsides, but cartilage damage persists (27). To examine this chronic aspect of AIA, we culled mice on days 7 and 28. In all genotypes, inflammatory synovitis and exudate decreased significantly between day 7 and day $28(P<0.001$ for all comparisons; data not shown). Aggrecan loss from wild-type and Chloe cartilage did not improve between day 7 and day 28 , even though inflammation was significantly resolved by day 28 . However, Jaffa cartilage was protected against aggrecan loss at day 28. Aggrecan loss in Jaffa cartilage was significantly less at day 28 than at day 7 $(P=0.005)$, and Jaffa cartilage at day 28 was significantly less than wild-type cartilage at day $28(P=0.001$; Figure $6 \mathrm{~B})$.

The most striking and important differences seen in the AIA model were in cartilage erosion (Figure 6C). Mild erosion was seen in all genotypes by day 7 . By day 28 , cartilage erosion had worsened

\section{Figure 6}

AIA in wild-type and aggrecan knockin mice. Mean \pm SEM of AIA scores for (A) aggrecan loss at days 1, 3, and 5 ( $n=5$ per group); (B) aggrecan loss at days 7 ( $n=35$ [wild-type], 15 [Chloe], 32 [Jaffa]) and 28 ( $n=49$ [wild-type], 19 [Chloe], 21 [Jaffa]); and (C) cartilage erosion at days 7 and 28 ( $n$ as in B) in wild-type (white bars), Chloe (black bars), and Jaffa (gray bars) mice. ${ }^{*} P<0.05$; ${ }^{* *} P<0.01$.

in wild-type (37\% increase) and Chloe (86\% increase), but had not progressed further in Jaffa (10\% decrease). Thus cartilage erosion in Jaffa mice at day 28 was significantly less than that in wild-type $(P=0.03)$ and Chloe mice $(P=0.005$; Figure $6 C)$, indicating that the Jaffa mouse is protected against progressive cartilage damage in this model.

The progressive erosion seen in wild-type cartilage between days 7 and 28 (Figure 6C) was associated with a decreased proportion of TUNEL-positive cells (49\% at day 7 compared with $9 \%$ at day 28 ) and an increased proportion of empty lacunae in noncalcified cartilage (20\% at day 7 compared with $77 \%$ at day 28 ), indicating progressive cell death in wild-type cartilage. Conversely, the proportions of TUNEL-positive cells and empty lacunae in Jaffa cartilage did not change between days 7 and 28; the proportion of empty lacunae in Jaffa cartilage was $28 \%$ on day 7 and $42 \%$ on day 28. Because Jaffa cartilage had less aggrecan loss, less cartilage erosion, and less empty chondrocyte lacunae, the TUNEL staining that persisted in Jaffa (44\% at day 7 compared with $39 \%$ at day 28 ) might reflect DNA repair (28) leading to improved matrix repair and cell survival.

Jaffa cartilage attempts to repair. Many joints from the AIA model examined at day 28 were completely devoid of toluidine blue staining in the noncalcified articular cartilage (Figure 7, A and D, asterisks). This suggests that the extensive aggrecan loss incurred in AIA by day 7 was maintained, a result either of lack of aggrecan synthesis or of failure to retain newly synthesized aggrecan. However, in other joints examined at the same time point, there was evidence of marked toluidine blue staining in pericellular regions (Figure 7C, arrowheads) as well as areas of more extensive staining into the interterritorial matrix (Figure 7, E and F). Areas of restored aggrecan staining were most prominent in femoral, rather than tibial, cartilage (Figure 7, B, C, E, and F). This result is strongly indicative of an attempt at repair, because at day 7 , joints from mice in all genotypes showed widespread lack of toluidine blue staining with little or no pericellular or interterritorial staining.

When restoration of cartilage aggrecan (i.e., repair) was quantitated from AIA histological sections, there was a marked improvement in Jaffa mice (12\%; Figure $7 \mathrm{G}$ ), but no improvement in Chloe or wild-type mice. The repair score in Jaffa mice was significantly different from that of both wild-type and Chloe mice $(P<0.05)$ and suggests that blocking aggrecanolysis at the IGD may potentiate cartilage repair.

\section{Discussion}

In this study we have characterized, and challenged, genetically modified mice whose aggrecan is resistant to proteolysis by aggrecanases in the IGD. The mutation was verified by Southern blotting, sequencing, and restriction enzyme digestion, and the consequent changes to the amino acid sequence were confirmed by analysis of aggrecan catabolites. The VTEGE ${ }^{373}$ neoepitope, used as a marker of aggrecanase cleavage in the IGD, was not detected in 

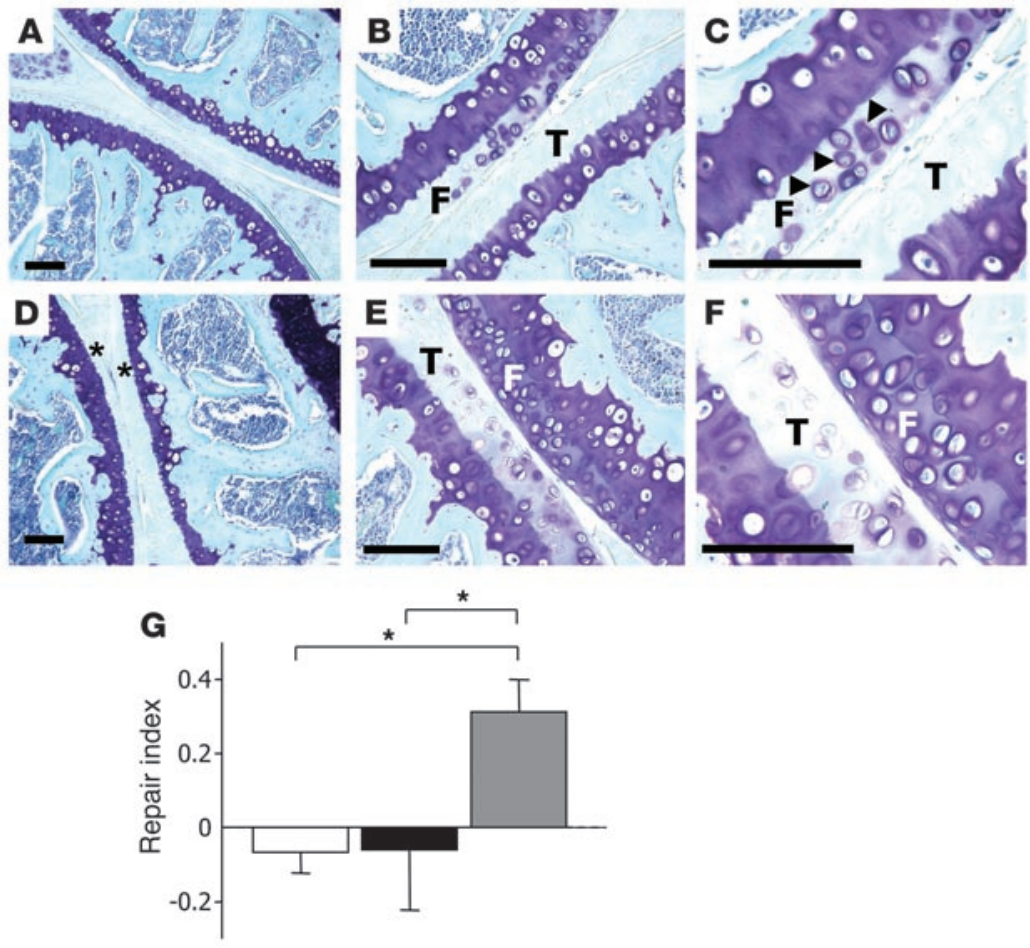

\section{Figure 7}

Cartilage repair in Jaffa mice. (A-F) Representative toluidine blue-stained sections of AIA joints from wildtype $(\mathbf{A}-\mathbf{C})$ or Jaffa (D-F) cartilage at day $\mathbf{2 8}$. A and $\mathbf{D}$ show no evidence of repair at day 28 . Note the complete loss of toluidine blue in the articular cartilage (asterisks). Repair in wild-type (B and $\mathbf{C}$ ) and Jaffa mice ( $\mathbf{E}$ and F) at day 28 was seen predominantly in the femur. F, femoral surface; $\mathrm{T}$, tibial surface. Arrowheads in $\mathbf{C}$ point to pericellular staining. Images in $\mathbf{C}$ and $\mathbf{F}$ are higher magnifications of the same sections shown in $\mathbf{B}$ and E. Scale bars: $100 \mu \mathrm{m}$. (G) Repair index, calculated as improvement in cartilage aggrecan loss score between days 7 and 28, in wild-type ( $n=49$; white bar), Chloe ( $n=19$; black bar), and Jaffa ( $n=21$; gray bar) femorotibial sections. Results are mean \pm SEM. ${ }^{\star} P<0.05$. direct extracts of Jaffa cartilage, nor was it possible to generate this epitope ex vivo by ADAMTS-5 digestion of aggrecan. In addition to the aggrecanases, MMP-8 $(29,30)$ and MMP-14 (31) can generate the ITEGE ${ }^{373}$ neoepitope in vitro. The absence of the VTEGE ${ }^{373}$ fragment we observed in Jaffa mice indicates that mutant aggrecan is not susceptible to cleavage at VTEGE ${ }^{373} \downarrow^{374} \mathrm{NVYSV}$ by aggrecanases, MMP-8, MMP-14, or other proteinases in vivo.

The Jaffa mice developed normally with no detectable skeletal or growth plate abnormalities, consistent with previous analyses of ADAMTS-1-, ADAMTS-4-, and ADAMTS-5-deficient mice, which also lack a skeletal phenotype $(11,12,26,32)$. Similarly, mice resistant to MMP cleavage in the IGD develop normally, with no skeletal deformities (25). Despite expression of ADAMTS-1 $(25,32)$ and ADAMTS-4 $(11,26,33)$ in the mouse growth plate and immunodetection of ITEGE ${ }^{373}$ neoepitope in rat $(33,34)$ and VTEGE ${ }^{373}$ neoepitope in mouse growth plate $(11,26)$, the phenotype of the unchallenged Jaffa mouse suggests that aggrecanases are not essential for aggrecan turnover during endochondral ossification. Collectively, our results from the present and previous studies $(12,25,32)$ strongly indicate that proteolysis by aggrecanases or MMPs in the IGD is not required for growth plate remodeling in development.

Other proteinases that might have a role in growth plate aggrecanolysis are m-calpain and the cathepsins. The calcium-dependent cysteine proteinase $\mathrm{m}$-calpain cleaves aggrecan in vitro (35, $36)$, and fragments consistent with m-calpain cleavage are present in extracts of mature bovine cartilage (36). Whether m-calpain is involved in growth plate aggrecanolysis in vivo has not been investigated. Several cathepsins are expressed by hypertrophic chondrocytes $(37,38)$, and in vitro, cathepsins B and D cleave at $\mathrm{G}^{344} \downarrow^{345} \mathrm{~V}(39,40)$ and $\mathrm{F}^{342} \downarrow^{343} \mathrm{~F}(41)$, respectively, in the aggrecan IGD. Fragments derived from cathepsin cleavage sites have not been detected, and their in vivo relevance has therefore not been confirmed. Cathepsin L-deficient mice have impaired bone development (42); however, the chondrocytic zones of the growth plate are indistinguishable between wild-type and mutant mice, and the deficiency is therefore thought to arise from abnormalities in osteoclast number and function.

In addition to proteinases, there may be nonproteolytic mechanisms for aggrecanolysis in cartilage (43), particularly in epiphyseal cartilages $(44,45)$. Hyaluronidases that depolymerize hyaluronan also effectively depolymerize the aggrecan aggregate. This is consistent with early electron microscopy studies reporting that aggregate size is reduced in calcifying (late hypertrophic) cartilage $(46,47)$. It is also consistent with recent studies that have identified release of intact, large-molecular weight aggrecan from fetal cartilage, with no evidence of IGD cleavage (45). Sztrolovics et al. have shown that hyaluronan released from bovine fetal cartilage explants following stimulation with interleukin- $1 \beta$ or retinoic acid is smaller than the hyaluronan released from unstimulated cultures (43). These results suggest that when fetal chondrocytes are stimulated to degrade their matrix, they do so partly by depolymerizing hyaluronan. The precise mechanisms within the growth plate are not known, but hyaluronan can be degraded by extracellular hyaluronidases (48) and by reactive oxygen radicals (49).

ADAMTS-5-deficient mice are protected against aggrecan loss and cartilage erosion in experimental arthritis $(11,12)$. ADAMTS-5 cleaves preferentially at several sites in the CS-2 domain $(15,17)$, and CS-2 sites as well as IGD sites are thought to be blocked in ADAMTS-5-deficient mice. IGD cleavage would liberate fragments carrying all the CS chains and is therefore thought to have the most detrimental effects on cartilage function; however, this has not been proved in vivo. Based on the consensus Ser-Gly dipeptide motif for CS attachment, $51 \%$ or $39 \%$ of CS chains would be lost by cleavage at the most $\mathrm{N}$-terminal site in the CS-2 domain in mice (SELE ${ }^{1279} \downarrow{ }^{1280}$ GRGT; Figure 2A) or humans (SELE ${ }^{1545} \downarrow$ ${ }^{1546}$ GRGT), respectively, if cleavage was blocked in the IGD. We 
found that ablating IGD cleavage in Jaffa reduced aggrecan loss by approximately $41 \%$, consistent with this estimation and with the fact that CS chains in the CS-1 domain (Figure 2A) are not lost in the absence of IGD cleavage.

The present study has examined the in vivo consequences of blocking ADAMTS cleavage at the IGD site only; we found that blocking cleavage at this site was sufficient to confer significant protection against aggrecan loss and cartilage erosion. At this point in time it is not possible to directly compare arthritis models of ADAMTS-5-deficient mice and Jaffa mice because the animals are on different genetic backgrounds. Genetic background has a profound effect on susceptibility to experimental arthritis (50-52), and we are presently backcrossing our mouse lines to identical genetic backgrounds in order to make direct comparisons in the future. Nevertheless, the present findings demonstrate that it was not necessary to block aggrecanase cleavage in the CS- 2 domain in order to achieve a degree of cartilage protection equivalent to that previously published for the ADAMTS-5-deficient mice (more than $50 \%$ reduction in histological score; ref. 11). Thus, substrate analogs with tight specificity for the IGD may be useful therapeutics for preventing cartilage damage.

Because ADAMTS- 4 and -5 are expressed in most tissues (12), we expect them to have substrates other than the cartilage-specific aggrecan. Both enzymes cleave the related proteoglycans versican and brevican. ADAMTS-4 cleaves cartilage oligomeric matrix protein (COMP; ref. 53) and biglycan (54). ADAMTS-5 also cleaves biglycan (54), but does not cleave COMP (53), fibromodulin, thrombospondin, or collagen types I or II (16) in vitro, but it would not be surprising if there were other cartilage substrates still to be identified. Thus, another important outcome of our study is confirmation that the in vivo protection conferred in the ADAMTS-5-deficient mice was most likely due to the loss of activity against aggrecan, rather than a loss of activity against 1 or more other cartilage molecules.

To evaluate cartilage damage in noninflammatory arthritis, we used DMM as a model of osteoarthritis-like disease. Aggrecan loss and cartilage damage in the tibial plateau was significantly reduced in Jaffa mice compared with wild-type and Chloe mice. This result is consistent with the protective effect seen in the same model in ADAMTS-5-deficient mice (11) and confirms that the chondroprotection observed in the previous study was primarily a result of inhibition of aggrecan cleavage. The most significant differences between wild-type and Jaffa mice were in the sum of scores rather than the severity of the worst lesion, which suggests that whereas focal cartilage damage induced by altered biomechanics still occurs in Jaffa mice, spread of the osteoarthritis-like pathology in the cartilage is curtailed.

In AIA, there was protection against early aggrecan loss during the onset of inflammation (days 1-5), indicating that aggrecanases have a significant role in this phase of the disease. Thereafter, aggrecan loss progressed in all genotypes until day 7 , when the extent of inflammation was maximal and the extent of aggrecan loss was the same in each genotype. It is likely that other proteinases including MMPs, neutrophil enzymes, and oxygen-derived free radicals play an increasingly important role as inflammation escalates. By day 7, any protection conferred in Jaffa is likely to be overwhelmed by these alternative mechanisms. Conversely, as inflammation subsides, aggrecanase activity may resume prominence relative to other activities, so that by day 28 aggrecan loss in wild-type and Chloe cartilage is further increased compared with
Jaffa cartilage, which begins instead to show signs of matrix repair and aggrecan accumulation.

Cartilage damage in the AIA model is progressive and irreparable (27). We have scored cartilage repair using a method that reflects the percent improvement in aggrecan accumulation at day 28 following total loss of aggrecan from noncalcified cartilage at day 7. The scores for wild-type mice showed that they failed to improve, which supports the previous finding of Stoop et al. that there is no significant cartilage repair in this model (27). However, our results with Jaffa mice showed that inhibiting aggrecanase cleavage in the IGD led to a $12 \%$ increase in the repair score at day 28 . We have yet to explore the precise mechanism of how this is achieved in vivo, but it is likely to reflect the imbalance between aggrecan synthesis/retention and aggrecan degradation. In Jaffa mice, the balance is most likely tipped in favor of retention because aggrecanolysis is reduced. When Jaffa chondrocytes commence new matrix synthesis, aggrecan may accumulate faster because the extent of loss was not as great as it was in wild-type cartilage. It is possible that all genotypes achieve some level of repair given sufficient time, but the rate at which repair is initiated and then continued might be greater in Jaffa mice. Other studies on the changing composition of cartilage matrix found that whereas large aggrecan fragments have a tissue half-life of approximately 3 years, the free $\mathrm{N}$-terminal globular domain of aggrecan, with no CS chains, survives in cartilage with an extraordinary long half-life of $20-25$ years $(55,56)$. It is therefore possible that partly degraded Jaffa aggrecan, with its reduced number of CS chains, has a longer half-life than wild-type aggrecan and thus accumulates more quickly. Furthermore, chondrocytes respond to mechanical deformation by upregulating expression of proteinases $(57,58)$. Because aggrecan is replaced pericellularly, it is possible that partly degraded Jaffa aggrecan accumulates more quickly than fully degraded wild-type aggrecan and that Jaffa cartilage may consequently be more effective at reestablishing a pericellular matrix that buffers chondrocytes against mechanically transduced signals that promote proteinase expression.

Finally, because aggrecan is thought to protect cartilage collagen from proteolysis (59), the Jaffa mutation may confer protection against both aggrecan loss and collagen II degradation. Reducing both aggrecan and collagen resorption might contribute to the reduced erosion seen in Jaffa mice and potentiate longer-term cartilage repair. In future experiments we will extend the length of the recovery period to determine whether there is even greater potential for cartilage repair in Jaffa mice with time.

Our results demonstrated that inhibiting aggrecanase cleavage in the IGD alone, without inhibiting aggrecanase cleavage in the CS-2 domain, was sufficient to significantly protect against cartilage degradation in both inflammatory and noninflammatory arthritis. Blocking aggrecanase cleavage in the IGD delayed aggrecanolysis in the acute phase of inflammatory arthritis and accelerated cartilage repair once inflammation subsided. Our results suggest that aggrecanase cleavage in the IGD is a critical determinant of cartilage pathology, whereas cleavage in the CS-2 domain might not contribute to acute cartilage pathology following injury or inflammation, but might instead be a component of C-terminal processing during normal maturation and aging (60).

\section{Methods}

Generation of mice resistant to aggrecanase cleavage in the aggrecan IGD. Heterozygous C57BL/6 aggrecan knockin mice with a targeted mutation in exon 7 were generated under contract by Ozgene and screened by Southern 
blot. We used sequencing and restriction digests to verify the mutation, which replaced nucleotides GCCCTGGGC with AACGTGTAC. The mutation changed amino acids ${ }^{374} \mathrm{ALGS}$ to ${ }^{374} \mathrm{NVYS}$, eliminating aggrecanase cleavage at the $\mathrm{E}^{373} \downarrow^{374} \mathrm{~A}$ bond in vitro (21) and in vivo. Mice homozygous for the mutation were designated Jaffa mice. In the present study, age-matched wild-type, Jaffa and heterozygous littermates were obtained from heterozygous breeding pairs to provide tissues for histology, cartilage extracts, and aggrecan analysis. Femoral head cartilage for in vitro culture experiments as well as animals for arthritis experiments were age-matched from homozygous wild-type or Jaffa breeding pairs. All mouse experiments were approved by the Animal Experimentation Ethics Committee of the Royal Children's Hospital.

Genotyping. The mutation in exon 7 introduced a unique AflIII restriction site. Genomic DNA was prepared by proteinase $\mathrm{K}$ digestion of tail biopsies, and a 758-bp cDNA fragment was amplified by PCR using forward and reverse primers $5^{\prime}$-AGGCGGAAATAGGAACTGA-3' and 5'-TCTCCAGTTCTCCTCAGC-3', respectively. Genotypes were determined by digesting the 758-bp PCR fragment with AflIII.

Aggrecan degradation in cartilage explant culture. Femoral head cartilage was harvested from young (2- to 4-week-old) wild-type and Jaffa mice under sterile conditions using forceps to induce a capital femoral physeal fracture as described previously (32). The femoral heads were cultured at $37^{\circ} \mathrm{C}$ for 3 days in HEPES-buffered DMEM containing 10\% FCS and then washed 3 times for 5 minutes each in serum-free DMEM. Individual femoral heads were cultured for 3 days in $0.4 \mathrm{ml}$ serum-free DMEM with or without $10 \mathrm{ng} / \mathrm{ml}$ recombinant human IL-1 $\alpha$ (Peprotech) with no medium changes, as described previously (32). At the end of the culture period, the media were collected and the femoral heads were blotted dry, weighed, and extracted for 48 hours at $4{ }^{\circ} \mathrm{C}$ with $4 \mathrm{M} \mathrm{GuHCl}$ containing proteinase inhibitors. The masses of sulfated glycosaminoglycans in the dialyzed extracts, culture media, and papain digests of the cartilage residue remaining after extraction were individually measured by 1,9-dimethylmethlene blue assay; the sum of the values represents the total aggrecan per sample. Differences in the release of aggrecan into the medium were expressed as a percentage of total aggrecan.

Aggrecan analysis. Mouse femoral heads were harvested and extracted for 48 hours at $4^{\circ} \mathrm{C}$ with buffered $4 \mathrm{M} \mathrm{GuHCl}$ containing proteinase inhibitors. The tissue residue remaining after extraction was digested with papain. Aggrecan content was determined by measuring the concentration of sulfated glycosaminoglycans in the dialyzed extracts and papain digests using the 1,9-dimethylmethylene blue assay (61).

Extracts of femoral head cartilage were analyzed by Western blotting with antibodies that recognize MMP or aggrecanase neoepitopes. Dialyzed extracts were buffered in $0.1 \mathrm{M}$ Tris acetate ( $\mathrm{pH}$ 6.5) and digested with 0.01 $\mathrm{U}$ chondroitinase $\mathrm{ABC}$ per $10 \mu \mathrm{g}$ glycosaminoglycan. Aliquots proportional to equal amounts of tissue weight were electrophoresed on SDS gels under reducing conditions and transferred to PVDF membrane (Millipore and Osmonics) for Western blotting.

Neoepitope antibodies recognize the newly created $\mathrm{N}$ or $\mathrm{C}$ termini of proteolytic fragments, but fail to recognize the same sequence of amino acids present in the intact protein. Neoepitopes are therefore markers of cleavage by specific families of proteinases. The rabbit polyclonal neoepitope antibodies used in this study were anti-ITEGE ${ }^{373}(62)$ and anti${ }^{374} \mathrm{ALGSV}$ (12), which recognize the products of aggrecanase cleavage in the IGD, and anti-DIPEN ${ }^{341}$, which recognizes a product of MMP cleavage in the IGD (24). The anti-ITEGE ${ }^{373}$ antibody was immunoreactive with the mouse sequence containing valine in place of isoleucine at position 369 $\left({ }^{369} \mathrm{VTEGE}^{373}\right)$. HRP-conjugated secondary antibodies were from Dako, and enhanced chemiluminescence kits were from Amersham.

The glycosaminoglycan composition of extracted aggrecan was analyzed by fluorophore-assisted carbohydrate electrophoresis as described previ- ously (63). Briefly, femoral heads were digested overnight with proteinase $\mathrm{K}$ at $60^{\circ} \mathrm{C}$. After digestion with chondroitinase $\mathrm{ABC}, \Delta$-disaccharides were collected in the filtrate of MicroCon 3 filter devices (Millipore), freeze-dried, and resuspended in $5 \mu \mathrm{l}$ of the fluorophore aminoacridone. Aliquots of standard CS $\Delta$-disaccharides (Seikagaku Corp.) were prepared at the same time. The samples and standards were incubated at room temperature for 15 minutes, after which $5 \mu \mathrm{l}$ of $1 \mathrm{M}$ cyanoborohydride was added, and fluorotagging proceeded for 16 hours at $37^{\circ} \mathrm{C}$. The samples were analyzed by electrophoresis on high-percentage polyacrylamide Monogels (Prozyme) or stored at $-70^{\circ} \mathrm{C}$. Fluorescently labeled $\Delta$-disaccharides were visualized on a Bio-Rad Chemidoc XRS system, and the mass of each fluorotagged species was quantitated using Quantity One software (version 4.5.0; Bio-Rad).

ADAMTS-5 digestion of wild-type and Jaffa aggrecans. Aggrecan present in dialyzed extracts of femoral head cartilage was incubated overnight at $37^{\circ} \mathrm{C}$ with $16 \mathrm{ng} / \mu \mathrm{l}$ ADAMTS-5 (Chemicon) in buffer containing $50 \mathrm{mM}$ Tris$\mathrm{HCl}$ ( $\mathrm{pH}$ 7.5), $100 \mathrm{mM} \mathrm{NaCl}$, and $10 \mathrm{mM} \mathrm{CaCl}_{2}$. Degradation products were immunodetected by Western blotting with neoepitope antibodies. After detecting neoepitopes, the blots were stripped (ReBlot Plus; Chemicon) and reprobed with monoclonal antibody 2B6 to confirm sample loading. Antibody $2 \mathrm{~B} 6$ recognizes terminal unsaturated $\Delta$ di-4S (64).

Mouse model of noninflammatory osteoarthritis. A unilateral model of noninflammatory osteoarthritis (DMM) was induced by microsurgical transection of the anterior medial meniscotibial ligament in the right knee of 8- to 10 -week-old mice, as described previously $(11,26)$. Briefly, the ligament was exposed through a medial parapatellar arthrotomy with the aid of a dissecting microscope. The patella was luxated laterally, the anterior fat pad dissected, and the ligament elevated and severed using curved dissecting forceps. Complete ligament transection was confirmed by the ability to manually displace the medial meniscus. Bleeding was controlled throughout surgery, and the cartilage was kept moist with sterile saline. After repositioning the patella, the joint was flushed with sterile saline and the incision closed in 3 layers with continuous $8 / 0$ vicryl in the joint capsule, continuous $8 / 0$ vicryl subcutaneously, and tissue glue for the skin. Animals were housed in groups and allowed unrestricted access to an exercise wheel 3 days after surgery.

Mice were culled 4 or 8 weeks after surgery, and their right knees were processed for histology. Studies have demonstrated no difference between unoperated and sham-operated knees and no changes in the lateral joint compartment following DMM in wild-type C57BL/6 mice (C.B. Little, unpublished observations). Serial sagittal $6-\mu \mathrm{m}$ sections spanning the entire width of the medial femorotibial joint were cut and mounted ( $3 \mathrm{sec}-$ tions per slide), and every third slide was stained with toluidine blue/fast green. A previously published scoring system $(11,26)$ was modified to separately score structural damage $(0$, normal cartilage; 1 , roughened articular surface and small fibrillations; 2 , fibrillation to immediately below the superficial layer or some loss of surface lamina; 3 , horizontal cracks/separations between calcified and noncalcified cartilage or fibrillation/clefts to calcified cartilage but no loss of noncalcified cartilage; 4 , mild loss of noncalcified cartilage [ $<10 \%$ surface area]; 5 , moderate loss of noncalcified cartilage [ $10 \%-50 \%$ surface area]; 6 , severe loss of noncalcified cartilage [ $>50 \%$ surface area]; 7 , erosion of cartilage to subchondral bone [any percent of surface area]) and aggrecan loss ( 0 , normal cartilage; 1 , decreased but not complete loss of toluidine blue staining in noncalcified cartilage; 2 , focal loss of toluidine blue staining in noncalcified cartilage [ $<30 \%$ surface area]; 3 , diffuse loss of toluidine blue staining in noncalcified cartilage [>30\% surface area]). The mean of (a) the worst scores and (b) the sum of scores (sum of all sections in which a positive score was noted) from 2 observers blinded to genotype was calculated. The cumulative score takes into account the surface area of cartilage affected in the joint $(11,26)$. Data were analyzed using Mann-Whitney $U$ test for unpaired nonparametric data. The proportion of apoptotic cells in representative sections from wild-type 
and Jaffa mice was determined by TUNEL staining (ApopTag; Chemicon) according to the manufacturer's instructions. TUNEL-positive cells were expressed as a percentage of total (TUNEL-positive plus TUNEL-negative plus empty lacunae) in the noncalcified cartilage of the femur and tibia.

Mouse model of AIA. AIA was induced in knee joints of 8- to 9-week-old mice using the AIA model as described previously $(12,32,65)$. Briefly, animals received an intradermal injection of $100 \mu \mathrm{g}$ methylated BSA (mBSA) in $100 \mu \mathrm{l}$ of Freund's complete adjuvant at 2 sites near the base of the tail on day 0 and day 14 . After 7-10 days, intra-articular mBSA (10 $\mu$ l of 20 $\mathrm{mg} / \mathrm{ml} \mathrm{mBSA}$ in $0.9 \%$ sterile saline or vehicle alone) was administered into the left and right knees of the mice. Animals were culled at 1, 3, 5, 7, and 28 days after intra-articular injection, and their knee joints were processed and stained with toluidine blue/fast green. Sagittal $6-\mu \mathrm{m}$ sections through the central weight-bearing region of the medial femorotibial joint were scored by 4 observers blinded to the treatment and genotype. Sections were scored for synovitis (0-3), synovial exudate (0-3), aggrecan loss (0-3), cartilage erosion (0-3), and bone erosion (0-3). The means of the 4 observers' scores for each section were calculated and compared using Mann-Whitney $U$ test for unpaired nonparametric data. The proportion of apoptotic cells in representative sections from wild-type and Jaffa mice was determined by TUNEL staining as described above.

Quantitating cartilage repair in inflammatory arthritis. To quantitate cartilage repair in the AIA model, the individual day-28 aggrecan loss score for each mouse was divided by the mean score for day- 7 aggrecan loss for the appropriate genotype. The cartilage repair score at day 28 was calculated by subtracting the number from 100: positive scores indicate percent improvement, and negative scores indicate the percent by which the score worsened, compared with day 7 .

1. Sandy, J.D., Neame, P.J., Boynton, R.E., and Flannery, C.R. 1991. Catabolism of aggrecan in cartilage explants. Identification of a major cleavage site within the interglobular domain. J. Biol. Chem. 266:8683-8685.

2. Loulakis, P., Shrikhande, A., Davis, G., and Maniglia, C.A. 1992. N-terminal sequence of proteoglycan fragments isolated from medium of interleukin-1-treated articular-cartilage cultures. Putative site(s) of enzymic cleavage. Biochem. J. 284:589-593.

3. Ilic, M.Z., Handley, C.J., Robinson, H.C., and Mok, M.T. 1992. Mechanism of catabolism of aggrecan by articular cartilage. Arch. Biochem. Biophys. 294:115-122.

4. Sandy, J.D., Flannery, C.R., Neame, P.J., and Lohmander, L.S. 1992. The structure of aggrecan fragments in human synovial fluid. Evidence for the involvement in osteoarthritis of a novel proteinase which cleaves the Glu 373-Ala 374 bond of the interglobular domain. J. Clin. Invest. 89:1512-1516.

5. Lohmander, L.S., Neame, P.J., and Sandy, J.D. 1993. The structure of aggrecan fragments in human synovial fluid. Evidence that aggrecanase mediates cartilage degradation in inflammatory joint disease, joint injury, and osteoarthritis. Arthritis Rheum. 36:1214-1222.

6. Tortorella, M.D., et al. 1999. Purification and cloning of aggrecanase-1: a member of the ADAMTS family of proteins. Science. 284:1664-1666.

7. Abbaszade, I., et al. 1999. Cloning and characterization of ADAMTS11, an aggrecanase from the ADAMTS family. J. Biol. Chem. 274:23443-23450.

8. Nagase, H., and Kashiwagi, M. 2003. Aggrecanases and cartilage matrix degradation. Arthritis Res. Ther. 5:94-103.

9. Jones, G.C., and Riley, G.P. 2005. ADAMTS proteinases: a multi-domain, multi-functional family with roles in extracellular matrix turnover and arthritis. Arthritis Res. Ther. 7:160-169.

10. Porter, S., Clark, I.M., Kevorkian, L., and Edwards, D.R. 2005. The ADAMTS metalloproteinases. Bio-

Statistics. Differences in the release of aggrecan into the medium associated with genotype and treatment were assessed using 2-factor ANOVA and Fisher's post-hoc analysis. Unpaired nonparametric data for parameters scored in the AIA and DMM models were analyzed using Mann-Whitney $U$ test. All data were analyzed using the StatView software package for Macintosh (StatView 5.0; SAS Institute Inc.). $P$ values of 0.05 or less were considered statistically significant.

\section{Acknowledgments}

This work was supported by the National Health and Medical Research Council (Australia) and the Arthritis Foundation of Australia Ulysses Club Fellowship (CBL). We are grateful for funding from Pfizer; for mouse husbandry support from Kerry Fowler and staff at the Murdoch Childrens Research Institute; and for constructive comments on the manuscript from Fraser Rogerson.

Received for publication October 26, 2006, and accepted in revised form March 13, 2007.

Address correspondence to: Amanda J. Fosang, Arthritis Research Group, University of Melbourne Department of Paediatrics and Murdoch Childrens Research Institute, Royal Children's Hospital, Flemington Road, Parkville 3052, Victoria, Australia. Phone: 61-3-83416466; Fax: 61-3-9345-7997; E-mail: amanda.fosang@mcri.edu.au.

Kate E. Lawlor's present address is: Department of Medicine, University of Cambridge School of Medicine, and Cambridge Institute for Medical Research, Cambridge, United Kingdom.

chem. J. 386:15-27.

11. Glasson, S.S., et al. 2005. Deletion of active ADAMTS5 prevents cartilage degradation in a murine model of osteoarthritis. Nature. 434:644-648.

12. Stanton, H., et al. 2005. ADAMTS5 is the major aggrecanase in mouse cartilage in vivo and in vitro. Nature. 434:648-652.

13. Fosang, A.J., Stanton, H., Little, C.B., and Atley, L.M. 2003. Neoepitopes as biomarkers of cartilage catabolism. Inflamm. Res. 52:277-282.

14. Flannery, C.R. 2006. MMPs and ADAMTs: functional studies. Front. Biosci. 11:544-569.

15. Tortorella, M.D., et al. 2000. Sites of aggrecan cleavage by recombinant human aggrecanase-1 (ADAMTS-4). J. Biol. Chem. 275:18566-18573.

16. Tortorella, M.D., Liu, R.Q., Burn, T., Newton, R.C., and Arner, E. 2002. Characterization of human aggrecanase 2 (ADAM-TS5): substrate specificity studies and comparison with aggrecanase 1 (ADAM-TS4). Matrix Biol. 21:499-511.

17. Sandy, J.D., Thompson, V., Doege, K., and Verscharen, C. 2000. The intermediates of aggrecanasedependent cleavage of aggrecan in rat chondrosarcoma cells treated with interleukin-1. Biochem. J. 351:161-166.

18. Patwari, P., Kurz, B., Sandy, J.D., and Grodzinsky, A.J. 2000. Mannosamine inhibits aggrecanasemediated changes in the physical properties and biochemical composition of articular cartilage. Arch. Biochem. Biophys. 374:79-85.

19. Cherney, R.J., et al. 2003. Potent and selective aggrecanase inhibitors containing cyclic $\mathrm{P} 1$ substituents. Bioorg. Med. Chem. Lett. 13:1297-1300.

20. Yao, W., et al. 2001. Design and synthesis of a series of (2r)-n(4)-hydroxy-2-(3-hydroxybenzyl)$\mathrm{n}(1)$ - [(1s,2r)-2-hydroxy-2,3-dihydro-1h-inden-1yl]butanediamide derivatives as potent, selective, and orally bioavailable aggrecanase inhibitors. J. Med. Chem. 44:3347-3350.

21. Mercuri, F.A., Maciewicz, R.A., Tart, J., Last, K., and Fosang, A.J. 2000. Mutations in the interglobular domain of aggrecan alter matrix metalloproteinase and aggrecanase cleavage patterns. Evidence that matrix metalloproteinase cleavage interferes with aggrecanase activity. J. Biol. Chem. 275:33038-33045.

22. Fosang, A.J., Last, K., Jackson, D.C., and Brown, L. 2001. Antibodies to MMP-cleaved aggrecan. In Matrix metalloproteinase protocols. I.M. Clark, editor. Humana Press. Totowa, New Jersey, USA. 425-449.

23. Fosang, A.J., Last, K., Gardiner, P., Jackson, D.C., and Brown, L. 1995. Development of a cleavage site-specific monoclonal antibody for detecting metalloproteinase-derived aggrecan fragments: detection of fragments in human synovial fluids. Biochem. J. 310:337-343.

24. Fosang, A.J., et al. 2000. Generation and novel distribution of matrix metalloproteinase-derived aggrecan fragments in porcine cartilage explants. J. Biol. Chem. 275:33027-33037.

25. Little, C.B., et al. 2005. Matrix metalloproteinases are not essential for aggrecan turnover during normal skeletal growth and development. Mol. Cell. Biol. 25:3388-3399.

26. Glasson, S.S., et al. 2004. Characterization of and osteoarthritis susceptibility in ADAMTS-4-knockout mice. Arthritis Rheum. 50:2547-2558.

27. Stoop, R., et al. 1999. Denaturation of type II collagen in articular cartilage in experimental murine arthritis. Evidence for collagen degradation in both reversible and irreversible cartilage damage. J. Pathol. 188:329-337.

28. Kanoh, M., et al. 1999. Significance of myocytes with positive DNA in situ nick end-labeling (TUNEL) in hearts with dilated cardiomyopathy: not apoptosis but DNA repair. Circulation. 99:2757-2764.

29. Arner, E.C., Decicco, C.P., Cherney, R., and Tortorella, M.D. 1997. Cleavage of native cartilage aggrecan by neutrophil collagenase (MMP-8) is distinct from endogenous cleavage by aggrecanase. J. Biol. Chem. 272:9294-9299.

30. Fosang, A.J., et al. 1994. Neutrophil collagenase (MMP-8) cleaves at the aggrecanase site E373-A374 
in the interglobular domain of cartilage aggrecan. Biochem. J. 304:347-351.

31. Buttner, F.H., et al. 1998. Membrane type 1 matrix metalloproteinase (MT1-MMP) cleaves the recombinant aggrecan substrate rAgg1mut at the 'aggrecanase' and the MMP sites. Characterization of MT1-MMP catabolic activities on the interglobular domain of aggrecan. Biochem. J. 333:159-165.

32. Little, C.B., et al. 2005. ADAMTS-1-knockout mice do not exhibit abnormalities in aggrecan turnover in vitro or in vivo. Arthritis Rheum. 52:1461-1472.

33. Mort, J.S., Flannery, C.R., Makkerh, J., Krupa, J.C., and Lee, E.R. 2003. Use of anti-neoepitope antibodies for the analysis of degradative events in cartilage and the molecular basis for neoepitope specificity. Biochem. Soc. Symp. 70:107-114.

34. Lee, E.R., et al. 2001. Enzymes active in the areas undergoing cartilage resorption during the development of the secondary ossification center in the tibiae of rats ages 0-21 days: I. Two groups of proteinases cleave the core protein of aggrecan. Dev. Dyn. 222:52-70.

35. Shimizu, K., et al. 1991. Immunohistochemical and biochemical demonstration of calcium-dependent cysteine proteinase (calpain) in calcifying cartilage of rats. J. Orthop. Res. 9:26-36.

36. Oshita, H., et al. 2004. Mature bovine articular cartilage contains abundant aggrecan that is $\mathrm{C}$-terminally truncated at Ala719-Ala720, a site which is readily cleaved by m-calpain. Biochem. J. 382:253-259.

37. Nakase, T., et al. 2000. Immunohistochemical detection of cathepsin $\mathrm{D}, \mathrm{K}$, and $\mathrm{L}$ in the process of endochondral ossification in the human. Histochem. Cell Biol. 114:21-27.

38. Soderstrom, M., et al. 1999. Cathepsin expression during skeletal development. Biochim. Biophys. Acta. 1446:35-46.

39. Fosang, A.J., et al. 1992. The interglobular domain of cartilage aggrecan is cleaved by PUMP, gelatinases and cathepsin B. J. Biol. Chem. 267:1947019474.

40. Mort, J.S., Magny, M.C., and Lee, E.R. 1998. Cathep$\sin \mathrm{B}$ : an alternative protease for the generation of an aggrecan 'metalloproteinase' cleavage neoepitope. Biochem. J. 335:491-494.

41. Handley, C.J., et al. 2001. Cathepsin D cleaves aggrecan at unique sites within the interglobular domain and chondroitin sulfate attachment regions that are also cleaved when cartilage is maintained at acid pH. Matrix Biol. 20:543-553.

42. Potts, W., et al. 2004. Cathepsin L-deficient mice exhibit abnormal skin and bone development and show increased resistance to osteoporosis following ovariectomy. Int. J. Exp. Pathol. 85:85-96.

43. Sztrolovics, R., Recklies, A.D., Roughley, P.J., and Mort, J.S. 2002. Hyaluronate degradation as an alternative mechanism for proteoglycan release from cartilage during interleukin-1beta-stimulated catabolism. Biochem. J. 362:473-479.

44. Plaas, A.H.K., and Sandy, J.D. 1993. A cartilage explant system for studies on aggrecan structure, biosynthesis and catabolism in discrete zones of the mammalian growth plate. Matrix. 13:135-147.

45. Sztrolovics, R., White, R.J., Roughley, P.J., and Mort, J.S. 2002. The mechanism of aggrecan release from cartilage differs with tissue origin and the agent used to stimulate catabolism. Biochem. J. 362:465-472.

46. Campo, R.D., and Romano, J.E. 1986. Changes in cartilage proteoglycans associated with calcification. Calcif. Tissue Int. 39:175-184.

47. Buckwalter, J.A., Rosenberg, L.C., and Ungar, R. 1987. Changes in proteoglycan aggregates during cartilage mineralization. Calcif. Tissue Int. 41:228-236.

48. Flannery, C.R., Little, C.B., Hughes, C.E., and Caterson, B. 1998. Expression and activity of articular cartilage hyaluronidases. Biochem. Biophys. Res. Commun. 251:824-829.

49. Roberts, C.R., Roughley, P.J., and Mort, J.S. 1989. Degradation of human proteoglycan aggregate induced by hydrogen peroxide. Protein fragmentation, amino acid modification and hyaluronic acid cleavage. Biochem. J. 259:805-811.

50. Campbell, I.K., Hamilton, J.A., and Wicks, I.P. 2000. Collagen-induced arthritis in C57BL/6 (H2b) mice: new insights into an important disease model of rheumatoid arthritis. Eur. J. Immunol. 30:1568-1575

51. Wooley, P.H., Dillon, A.M., Luthra, H.S., Stuart, J.M., and David, C.S. 1983. Genetic control of type II collagen-induced arthritis in mice: factors influencing disease susceptibility and evidence for multiple MHC-associated gene control. Transplant. Proc. 15:180-185.

52. Glasson, S.S., et al. 2006. Evaluation of twelve knock-out mice following surgical induction of osteoarthritis. Paper no. 0216 presented at the 52nd Annual Meeting of the Orthopaedic Research Society. March 19-22. Chicago, Illinois, USA

53. Dickinson, S.C., et al. 2003. Cleavage of cartilage oligomeric matrix protein (thrombospondin-5) by matrix metalloproteinases and a disintegrin and metalloproteinase with thrombospondin motifs. Matrix Biol. 22:267-278.

54. Melching, L.I., Fisher, W.D., Lee, E.R., Mort, J.S., and Roughley, P.J. 2006. The cleavage of biglycan by aggrecanases. Osteoarthritis Cartilage. 14:1147-1154.

55. Maroudas, A., Bayliss, M.T., Uchitel-Kaushansky, N., Schneiderman, R., and Gilav, E. 1998. Aggrecan turnover in human articular cartilage: use of aspartic acid racemization as a marker of molecular age. Arch. Biochem. Biophys. 350:61-71.

56. Verzijl, N., et al. 2001. Age-related accumulation of the advanced glycation endproduct pentosidine in human articular cartilage aggrecan: the use of pentosidine levels as a quantitative measure of protein turnover. Matrix Biol. 20:409-417.

57. Fitzgerald, J.B., et al. 2004. Mechanical compression of cartilage explants induces multiple timedependent gene expression patterns and involves intracellular calcium and cyclic AMP. J. Biol. Chem. 279:19502-19511.

58. Fitzgerald, J.B., Jin, M., and Grodzinsky, A.J. 2006. Shear and compression differentially regulate clusters of functionally related temporal transcription patterns in cartilage tissue. J. Biol. Chem. 281:24095-24103.

59. Pratta, M.A., et al. 2003. Aggrecan protects cartilage collagen from proteolytic cleavage. J. Biol. Chem. 278:45539-45545.

60. Ilic, M.Z., Robinson, H.C., and Handley, C.J. 1998. Characterization of aggrecan retained and lost from the extracellular matrix of articular cartilage. Involvement of carboxyl-terminal processing in the catabolism of aggrecan. J. Biol. Chem. 273:17451-17458

61. Farndale, R.W., Sayers, C.A., and Barrett, A.J. 1982. A direct spectrophotometric microassay for sulfated glycosaminoglycans in cartilage cultures. Connect. Tissue Res. 9:247-248.

62. Mercuri, F.A., et al. 1999. Recombinant human aggrecan G1-G2 exhibits native binding properties and substrate specificity for matrix metalloproteinases and aggrecanase. J. Biol. Chem. 274:32387-32395.

63. Plaas, A.H.K., West, L., Midura, R.J., and Hascall, V.C. 2001. Disaccharide composition of hyaluronan and chondroitin/dermatan sulfate. Analysis with fluorophore-assisted carbohydrate electrophoresis. Methods Mol. Biol. 171:117-128.

64. Couchman, J.R., Caterson, B., Christner, J.E., and Baker, J.R. 1984. Mapping by monoclonal antibody detection of glycosaminoglycans in connective tissues. Nature. 307:650-652

65. Brackertz, D., Mitchell, G.F., and Mackay, I.R. 1977. Antigen-induced arthritis in mice. I. Induction of arthritis in various strains of mice. Arthritis Rheum. 20:841-850. 